\title{
La conciencia lingüística en el aula de ELE: lengua artificial, lengua natural y diversidad sociolingüística
}

\author{
Patricia Fernández Martín \\ Universidad de Vigo \\ patriciafernandezmartin@gmail.com
}

Resumen: El objetivo de este artículo es defender la necesidad de hacer conscientes a los alumnos de español como segunda lengua de dos cuestiones que atañen a la ideología lingüística: por un lado, la distinción entre la lengua artificial (lengua estándar), que es el objeto de aprendizaje del extranjero, y la lengua natural (lengua oral), que es la herramienta de comunicación nativa; y por otro lado, la existencia de facto de una diversidad sociolingüística en España, invisible en los manuales, y cuyo desconocimiento da lugar a múltiples malentendidos. Para ello, comenzaremos en la primera sección analizando los conceptos de «lengua estándar» $y$ «dialecto» en el Marco común europeo de referencia para las lenguas, así como el de competencia sociolingüística, para comprender las bases metodológicas de este documento. Después, argumentaremos los motivos por los que se debería hacer lo que defendemos, es decir, concienciar al alumno tanto de la artificialidad de la lengua que aprende como de la diversidad lingüística española; y terminaremos, antes de las conclusiones, analizando críticamente una unidad didáctica con objetivos sociolingüísticos, que hemos llevado al aula con varios grupos de extranjeros, que vivían en Madrid y asistían a clases de EL2. La principal conclusión pasa por comprender que los estudiantes también tienen prejuicios y que sólo con una buena educación lingüística, apoyada en ejemplos de la vida cotidiana, pueden eliminarse.

Palabras clave: lengua artificial, lengua natural, sociolingüística 
Abstract: The aim of this paper is to defend the need to make students of Spanish as a second language aware of two issues concerning linguistic ideology. On the one hand, we make a difference between the artificial language (standard language), which is the learning object of the student, and the natural language (spoken language), which is the native tool of communication. On the other hand, we remark the existence de facto of a sociolinguistic diversity in Spain, notto-be-seen in the handbooks of Spanish as a Second Language, whose ignorance leads to multiple misunderstandings. Then, we will start the text analyzing the concepts of «standard language» and «dialect» in the Common European Framework of Reference for Languages, as well as the sociolinguistic competence, in order to understand the methodological basis of this document. After that, we argue why teachers should make students aware of the artificiality of language they learn, and of the Spanish linguistic diversity. Finally, we criticize a lesson with sociolinguistic objectives, that has been applied in several groups of foreigners living in Madrid and attending classes of Spanish. The main conclusion leads to understand that also students have prejudices and that only a good educational linguistics, supported by examples from everyday life, can remove them.

Key words: Artificial Language, Natural Language, Sociolinguistics. 


\section{Introducción}

El enfoque social en la enseñanza de lenguas implica entender el desarrollo del aprendizaje inserto dentro de un contexto en el que el código semiótico pasa a un segundo plano, y se focaliza la interrelación del hablante con los factores contextuales relevantes que permiten su desarrollo como agente social. De este modo, a la lengua objeto, elemento fundamental del aprendizaje en los métodos tradicionales (Richards y Rogers, 2003: 15-17; Sánchez, 1997: 133-141), a la que tal vez ya a partir del enfoque natural de los años 70 se le suma el importante papel del aprendiz (Zanón, 2007; Richards y Rogers, 2003: 178-180), conviene ahora añadir el contexto sociocultural en el que dicho proceso se produce, entendiendo por tal contexto el ambiente en que se desarrolla el estudio de la lengua (Moreno Fernández, 2007: 57-58; Caballero Murillo y Cuadrado Gordillo, 2007; Fernández Martín, 2008), lo que incluiría necesariamente el Centro, el docente y, naturalmente, el estudiante (Martínez-Otero, 2008; Fernández Martín, 2013a; 2013b).

Así, entendemos que nuestra investigación se encuentra inserta dentro de este marco teórico, ya que tratamos de defender la necesidad de adoptar dicho enfoque también desde una perspectiva explícita, que haga consciente al alumno de las ideologías que se esconden detrás de determinados prejuicios relacionados con la diversidad lingüística y con la lengua que está estudiando (Cots et alii, 2007), especialmente si se halla en situaciones de inmersión, en las que el contacto con hablantes de la lengua objeto resulta más probable, naturalmente, que si aprende la LE como una materia complementaria, fuera de sus territorios geolingüísticos (Moreno Fernández, 2007: 57-58).

De esta forma, por un lado, se pretende mejorar la comprensión del propio proceso de aprendizaje, por medio de la reflexión de las características sociales que rodean la construcción de la lengua objeto y, por otro lado, se busca evitar sensaciones de fracaso por ignorar la existencia de una diversidad lingüística española a la que raramente se hace alusión en los manuales (Fernández Martín, 2013c: 86-92).

En el primer caso, estamos refiriéndonos concretamente a la necesidad de hacer explícita la distinción entre lengua artificial (lengua estándar, lengua ideal, lengua aprendida) y lengua natural (lengua variable, lengua oral, lengua adquirida), de manera que el estudiante sea consciente de que cuando aprende una LE no está accediendo directamente a las variedades dialectales que se va a encontrar en sus interacciones orales cotidianas, sino que está estudiando una variedad lingüística igual de perfecta o imperfecta que cualquier otra, válida tan sólo para unos contextos determinados, que la dotan de ventaja social por encontrarse 
excesivamente valorada, al ser considerada la variedad de prestigio dentro de la comunidad lingüística, apoyada comúnmente por el grafocentrismo imperante y por la idea de que la educación escolar proporciona mejores accesos al mercado laboral (Ferreiro, 2002; Moreno Cabrera, 2004, 2005, 2008; Cots et alii, 2007; Campero, 2007; EACEA, 2011: 9 ss; Velasco y Díaz de Rada, 2006: 137-250; Mesthrie, 2008).

A este respecto, cabe justificar la equivalencia realizada entre lengua artificial, lengua estándar, lengua ideal, lengua aprendida, por un lado, y lengua natural, lengua variable, lengua oral, lengua adquirida y dialecto, por otro. Así, siguiendo a Moreno Cabrera (2008:55), entendemos que la lengua estándar o común es una «construcción ficticia que tiene realidad como un esquema o proyecto ideológico que se intenta llevar a la práctica de modo más o menos efectivo», es decir, la lengua estándar es una creación convencional, inexistente en la realidad (Moreno Cabrera, 2004: 51), porque no es adquirida (implícitamente) mediante la interacción esperable por (y en) el grupo social, sino aprendida (explícitamente) en situaciones de instrucción, pero como es fija y homogénea, funciona perfectamente como objeto de estudio para los alumnos extranjeros (y otros que no necesariamente lo son).

Por el contrario, entendemos que el concepto de «lengua natural» (la lengua adquirida sin instrucción alguna), resulta equivalente en la práctica a «dialecto», dado que ambos aluden a la variante lingüística que realmente se habla, no a la que empleamos como modelo idealizado de prestigio. En otras palabras, «nadie habla abstracciones» (Moreno Cabrera, 2004: 51) y la lengua estándar lo es, por lo que distinguir entre lo que realmente se habla (dialecto, lengua natural, lengua oral) y lo que, en opinión de algunos, se debe hablar (lengua estándar, lengua artificial, lengua escrita) resulta esencial para no caer en ideologías sociopolíticas que poco tienen que ver con lo lingüístico y que, sin embargo, al haberse propagado incluso entre los expertos (Moreno Cabrera, 2008: 174 ss), resultan útiles como herramientas de análisis (cfr. $§ 1.1)$.

En el segundo caso, la creación de la consciencia de la diversidad lingüística y cultural (Cots et alii, 2007) ha de servir al estudiante para hacerle igualmente saber que en un país plurilingüe como España no se habla solo español (Etxebarria, 2002). Esto, que puede parecer obvio pero, desgraciadamente, no lo es (Moreno Cabrera, 2014), puede implicar un sinfín de graves malentendidos comunicativos (falsas expectativas, incorrectas atribuciones y fracasos significativos) y un aumento de la frustración por el esfuerzo dedicado, si el estudiante llega a creer que las horas invertidas en el estudio de la LE no han sido suficientes para entender a todos los nativos del español. Especialmente, estas consecuencias resultan 
esperables para el estudiante extranjero al que se le ha ofrecido constantemente la idea (que no es falsa, pero sí incompleta, segmentada y parcial) de que la lengua española la hablan 250 millones de personas y que es, por ello, extremadamente útil estudiarla porque abarca numerosísimas posibilidades comunicativas (Moreno Cabrera, 2008: 155 ss).

Entonces, nos encontramos dos objetivos: defender la necesidad de hacer consciente al alumno de la (in)existencia de una lengua estándar de forma empírica y, sin embargo, de su utilidad como herramienta evaluadora y guía a seguir durante el proceso de aprendizaje; y la obviedad de hacer visibles en el aula de ELE (y mucho más especialmente en el aula de EL2) las otras lenguas de España, prácticamente inexistentes en los manuales utilizados habitualmente como libros de texto y, por deducción, de la práctica docente (Fernández Martín, 2013cः 86).

Para ello, comenzaremos en la primera sección analizando los conceptos de «lengua estándar» $\mathrm{y}$ «dialecto» (como equivalente, recordemos, a «lengua natural») en el Marco común europeo de referencia para las lenguas (Instituto Cervantes, 2002) ${ }^{1}$, así como la percepción que se deja entrever en el mismo documento acerca de la competencia sociolingüística ( $c f r . \S 1$ ); pasaremos después a argumentar los motivos por los que se debería hacer lo que defendemos, es decir, concienciar al alumno tanto de la artificialidad de la lengua que aprende como de la diversidad lingüística española (cfr. $\$ 2)$; y terminaremos, antes de las conclusiones, analizando críticamente una unidad didáctica con objetivos sociolingüísticos, que hemos llevado al aula con varios grupos de alumnos extranjeros que vivían en Madrid y necesitaban, por tanto, aprender español para comunicarse en su día a día (cfr. § 3$)$.

\section{El enfoque social de la enseñanza de lenguas en el Marco}

En este apartado procedemos a indagar cuál es la base metodológica que subyace al Marco en lo que aquí nos ocupa.

Para establecer una primera aproximación, hemos empleado una búsqueda automática de las palabras «dialecto» y «lengua estándar» en el documento del Marco común europeo de referencia para las lenguas (Instituto Cervantes, 2002), de manera que nos ofrezca una visión general de la ideología subyacente. Después, hemos hecho lo propio con las palabras «sociolingüística»y «sociolingüístico» $y$ sus variantes flexivas y derivadas.

1 Citamos de acuerdo con la versión española, realizada por el Instituto Cervantes y referenciada en la bibliografía final. 
Así, de la palabra «dialecto» hemos encontrado tres ocurrencias (Instituto Cervantes, 2002: 4, 116, 118), y su forma en plural, «dialectos», aparece en la página 4 , mientras que el derivado «dialectales» aparece dos veces en la 118 y una sola en la página 131.

Por lo que respecta al sintagma «lengua estándar», aparece en numerosas ocasiones (Instituto Cervantes, 2002: 26, 31, 37, 38, 69 [x3], 70 [x3], 73, 76, 77 [x2], 212, 224 [x7], 225, 226), a diferencia de «variedad estándar» que no se da en ningún caso. Asimismo, el adjetivo «estándar» complementa a otros sustantivos como «pronunciación» (p. 70), «acento» (p. 76 [x2]), «nivel» (pp. 78, 246), «formato» (p. 94), «parámetros o criterios» (p. 190) $)^{2}$ « «lengua hablada» (p. 224 [x2]), lo que deja entrever cuál es el objetivo real del aprendizaje del estudiante de lenguas extranjeras. En la próxima sección lo analizamos con más detalle (cfr. $\S 1.1$.

El término «sociolingüística» aparece en el Marco en numerosas ocasiones (Instituto Cervantes, 2002: 13, 28, 106, 116 [x3], 118 [x3], 119 [x2], 132, 137, 153 [x2], 154, 171, 179, 194 [x2], 195 [x3], 211, 212, 214 [x2]), de las cuales, en una se muestra como adverbio («sociolingüísticamente», p. 118). También se da en la forma masculina en algunos casos (Instituto Cervantes, 2002: 13, 14 [x2], 119, 134 [x3], 153 [x2], 156, 169 [x2], 172, 187). De aquí se puede deducir que parece existir cierta conciencia de la importancia del factor sociolingüístico en la enseñanza de idiomas (cfr. $\$ 1.2)$.

\section{1 «Dialecto» (lengua natural) y «lengua estándar» (lengua artificial)}

Comencemos, pues, analizando en qué contextos se da la palabra «dialecto». Cuando se da en términos globales, parece mostrarse una oposición entre este concepto y el de lengua, como se dice al inicio del documento ( «[...] los interlocutores pueden cambiar de una lengua o un dialecto a otro, explotando así la habilidad que tiene cada uno para expresarse en una lengua y para comprender otra», p.4), mientras que cuando aparece inserto dentro de la competencia sociolingüística (Instituto Cervantes, 2002: §5.2.25), se lo considera una característica natural del habla nativa, por la que el estudiante de LE puede llegar a conocer con mayor profundidad al hablante de la lengua que está estudiando («El reco-

2 En este caso, se está haciendo alusión a los criterios evaluadores sobre los propios descriptores, no a la lengua estándar en sí, como en los otros ejemplos aducidos, por lo que no lo mencionaremos más: «Las escalas de descriptores de los niveles comunes de referencia se pueden utilizar para proporcionar un conjunto de criterios definidos [...] o para describir los niveles de exigencia representados por criterios existentes en función de los niveles comunes. En el futuro, quizá se puedan proporcionar muestras o ejemplos de actuaciones típicas en distintos niveles comunes de referencia para contribuir a la creación de unos parámetros o criterios estándar» (Instituto Cervantes, 2002: 190). 
nocimiento de dichos rasgos dialectales ofrece, pues, claves significativas respecto a las características del interlocutor», p. 118), lo que implica, a nuestro juicio, una aceptación implícita de la inexistencia de la lengua estándar como variante lingüística empírica naturalmente adquirida, y convertida en un instrumento de aprendizaje, útil en la medida en que es aferrable para el alumno (y para el profesor), porque se puede sistematizar para enseñar y porque se presenta generalmente en contextos escritos (Moreno Cabrera, 2005; Ferreiro, 2002).

Así, en la siguiente afirmación parece reconocerse que el alumno accede, en sus interacciones sociales, a una forma de lengua que no va a coincidir por completo con la aprendida, como puede desprenderse del hecho de que se perciba la existencia de un segundo objeto de aprendizaje con el que el estudiante entrará en contacto a través de sus relaciones con nativos. En otras palabras, en el Marco se puede comprobar que el propio estudiante ha de aprender dos variantes lingüísticas, esto es, la artificial («lengua estándar») y la natural («rasgos dialectales»), a la vez que se familiariza con los valores socioantropológicos que la variante de su elección tiene en la comunidad de habla en que se encuentra:

Con el paso del tiempo, los alumnos también entrarán en contacto con hablantes de varias procedencias; antes de que puedan adoptar ellos mismos las formas dialectales, deberían ser conscientes de sus connotaciones sociales y de la necesidad de coherencia y de constancia.

(Instituto Cervantes, 2002: 118)

Asimismo, parece confirmar nuestra interpretación el hecho de que todos los casos contabilizados en que se da el sintagma «lengua estándar», aparezcan siempre insertos en algún tipo de descriptor o en afirmaciones que aluden a ellos, lo que permite leer entre líneas que se emplea este concepto para medir el objeto de estudio del estudiante de LE, y no necesariamente la herramienta de comunicación real de los hablantes nativos.

A este respecto, tomamos como ejemplo el siguiente párrafo que consideramos esclarecedor, en el cual se habla de dos de las características del nivel umbral (B1):

La primera es la capacidad de mantener una interacción y de hacerse entender en una variedad de situaciones; por ejemplo: generalmente comprende las ideas principales de los debates extensos que se dan a su alrededor siempre que el discurso se articule con claridad en nivel de lengua estándar; ofrece y pide opiniones personales en un debate informal con amigos [...].

(Instituto Cervantes, 2002: 36 [la cursiva es nuestra]) 
Por lo dicho anteriormente, creemos que no se está tomando como referencia la lengua estándar porque sea más fácil de aprender, porque sea común a más hablantes de la LE o porque, al tratarse de la variante generalmente escrita, sea más fácilmente abarcable desde la perspectiva didáctica, sino que pensamos que se hace alusión a ese nivel de lengua estándar, sencillamente, porque esta es la variante lingüística que se le enseña al estudiante durante su proceso de aprendizaje, por lo que sería incoherente pedir, a la hora de evaluar, que se desenvolviera, pongamos, en la variante andaluza, argentina o leonesa. En el Marco encontramos, pues, perfectamente la conciencia lingüística (Cots et alii, 2007) de la artificialidad de la variante estándar como variante real (Moreno Cabrera, 2008, 2014). Pero a la vez, se nos muestra también la utilidad de esta misma variante como objeto igualmente real de aprendizaje, necesario desde una perspectiva discente (y docente), al funcionar como patrón evaluador, válido especialmente en las situaciones prestigiosas en las que el extranjero, por no hablar con fluidez la lengua, siempre va a sentirse acomplejadamente inferior.

En otras palabras, podría ser contraproducente enseñarle una variante desprestigiada (distinto es que él decidiera aprenderla, naturalmente) porque esto añadiría a su vulnerabilidad lingüística la debilidad del prestigio social de la variante dialectal seleccionada. Y de esto, como vemos, se es perfectamente consciente en el Marco común europeo de referencia para las lenguas, como se demuestra en los otros sintagmas, ya aludidos, en los que aparece el concepto de estándar: «pronunciación» (p. 70), «acento» (p.76 [x2]), «nivel» (pp. 78, 246), «formato» (p. 94) y «lengua hablada» (p. 224 [x2]), todos ellos insertos en los descriptores de la lengua estándar que constituye el objeto de aprendizaje.

Por tanto, cabe pensar que, si bien en el aula hay que focalizar la enseñanza en la lengua estándar por ser la variante prestigiosa (Moreno Cabrera, 2004: 51), no sobra, creemos, resaltar la importancia de las demás variantes de una forma explícita (Mesthrie, 2008). Incluso se debería llegar a hacer entender al alumno que la variante que se está estudiando (la estándar) no tiene un correlato fuera del aula, aparte de su uso en ciertas situaciones comunicativas (académicas, profesionales), que entonces deben enseñarse como situaciones concretas en las que la lengua adquiere unos usos concretos, diferentes a los que muestra en otras situaciones menos artificiosas (Tusón y Calsamiglia, 1999). No hacer consciente de estas diferencias al estudiante puede llevarle a un claro sentimiento de fiasco, por creer que la variante que él está aprendiendo es exactamente la misma que se va a encontrar en sus interacciones con nativos, lo que, naturalmente, bien es sabido que no va a ocurrir. 


\subsection{La conciencia sociolingüística en el Marco}

Igualmente, parece clara la conciencia sociolingüística del documento que nos ocupa, fundamentalmente porque en él se defiende la competencia sociolingüística como una competencia más (Instituto Cervantes, 2002: $\S \$ 5.2 .2,6.4 .7$ ) que constituye, junto con las competencias lingüísticas (Instituto Cervantes, 2002: \$\$ $5.2 .1,6.4 .6)$ y pragmáticas (Instituto Cervantes, 2002: $₫ \$ 5.2 .3,6.4 .8$ ), las competencias comunicativas de la lengua (Instituto Cervantes, 2002: $\$ 5.2$ ), objetos de aprendizaje del estudiante.

Dentro de la competencia sociolingüística (Instituto Cervantes, 2002: $\S \S$ 5.2.2) se incluyen los marcadores lingüísticos de relaciones sociales (formas de tratamiento, convenciones para los turnos de palabra), las normas de cortesía (positiva o negativa, según el contexto), las expresiones de la sabiduría popular (refranes, modismos), las diferencias de registro (solemne, formal, informal, familiar) y el dialecto y el acento (clase social, procedencia regional, origen nacional, grupo étnico, grupo profesional).

Esta concepción del objeto de estudio conlleva, además, la idea de que en el nivel del habla no hay una oposición entre «correcto» o «incorrecto», típica de la consideración tradicional del aprendizaje (Instituto Cervantes, 2002: $₫ 6.5$; Richards y Rodgers, 2003; Sánchez, 2000; Zanón, 2007), sino entre «adecuado» o «inadecuado», lo que queda prístinamente plasmado en la concepción de adecuación sociolingüística que se menciona continuamente en los descriptores (p. 119):

Desde el nivel B2, se ve que los usuarios son capaces de expresarse adecuadamente en una lengua que es sociolingüísticamente apropiada a las situaciones y a las personas implicadas, y comienzan a adquirir la capacidad de abordar la variedad del habla, además de un grado mayor de control sobre el registro y los modismos.

(Instituto Cervantes, 2002: 118 [la cursiva es nuestra])

Por otra parte, dado que al comienzo de la descripción de la competencia sociolingüística se alude a su relación con los conocimientos socioculturales ( Como se destacó respecto a la competencia sociocultural, puesto que la lengua es un fenómeno sociocultural, gran parte de lo que contiene el Marco de referencia, respecto a este ámbito, resulta adecuado para la competencia sociolingüística», p. 116), merece la pena destacar algunos de los conocimientos ahí reflejados y que nos interesan especialmente (Instituto Cervantes, 2002: $\$ 5.1 .1 .2$ ), ya que se entiende que forman parte de la competencia general conformada por el conocimiento declarativo o saber: los valores, las creencias y las actitudes de la sociedad 
cuya lengua se estudia, respecto, entre otros, a las culturas regionales, la identidad nacional, las minorías y los países, estados y pueblos extranjeros (Instituto Cervantes, 2002: 100); y las convenciones sociales y las relaciones personales (Instituto Cervantes, 2002: 101) que están regidas, entre otros factores, por la lengua que se emplea (López Morales, 1989; Heller, 1982; Jupp et alii, 1982; Chambers, 1995; Fasold, 1996; Duranti, 2000; Vinagre, 2005).

Si a esto se le añade la base metodológica subyacente en el Marco, el enfoque orientado a la acción (Instituto Cervantes, 2002: § 2.1), entendido como aquel que defiende el uso de la lengua en situaciones sociales reales que otorgan al hablante todo tipo de etiquetas sociocognitivas, a la vez que le permiten realizar las tareas necesarias para vivir en el día a día, consideramos este enfoque propicio para nuestros propósitos:

El enfoque aquí adoptado, en sentido general, se centra en la acción en la medida en que considera a los usuarios y alumnos que aprenden una lengua principalmente como agentes sociales, es decir, como miembros de una sociedad que tiene tareas (no sólo relacionadas con la lengua) que llevar a cabo en una serie determinada de circunstancias, en un entorno específico y dentro de un campo de acción concreto [... ] Hablamos de «tareas» en la medida en que las acciones las realizan uno o más individuos utilizando estratégicamente sus competencias específicas para conseguir un resultado concreto.

(Instituto Cervantes, 2002:9)

Y lo consideramos adecuado (Fernández Martín, 2013c: 73), en primer lugar, porque se centra en el uso real de la lengua más allá del código («considera a los usuarios y alumnos que aprenden una lengua principalmente como agentes sociales»), lo que implica, en segundo lugar, entender el idioma como vehículo de relaciones y expresión de identidades que siempre traspasa lo estrictamente dicho ( «estas actividades forman parte de un contexto social más amplio, que por sí solo puede otorgarles pleno sentido») y porque, finalmente, la realización de las tareas (de aula) se puede relacionar con el éxito comunicativo (en el mundo real) («Hablamos de «tareas» en la medida en que las acciones las realizan uno o más individuos utilizando estratégicamente sus competencias específicas para conseguir un resultado concreto»).

Todo ello supone, por un lado, una forma de evaluar no sólo el proceso de enseñanza/aprendizaje de la lengua artificial (si el usuario desempeña correctamente las tareas en el aula, entonces ha conseguido el nivel pretendido, como se señala en los descriptores del Marco), sino también la misma capacidad del alumno de desenvolverse en la lengua extranjera estudiada para resolver sus necesidades 
comunicativas concretas en la lengua natural ("en una serie determinada de circunstancias, en un entorno específico y dentro de un campo de acción concreto»).

Como consecuencia, la visibilización de las otras lenguas en la clase de ELE se hace urgentemente necesaria para alcanzar dicho éxito comunicativo, dado el claro apoyo metodológico con que cuenta en el Marco, especialmente si tenemos presente que las lenguas de España existen desde una perspectiva empírica (Etxebarria, 2002; Moreno Cabrera, 2014), es decir, forman parte del bagaje sociocultural e identitario español. Asimismo, dado que su desconocimiento puede inducir a malentendidos comunicativos, empezando por el sentimiento de fracaso del estudiante de ELE (que puede pensar que no ha sido suficiente el esfuerzo dedicado al aprendizaje del español), y terminando por sus críticas e improperios hacia los hablantes bilingües (considerados maleducados por hablarle en una lengua que él no entiende), no conocerlas, al menos desde una perspectiva teórica, puede frustrar su proceso de aprendizaje y destruir radicalmente cualquier intento de comunicación que se haya propuesto, tirando así por la borda el espíritu que subyace al enfoque orientado a la acción del Marco.

\section{Hacia la visibilización lingüística}

Vemos, por tanto, cómo en el Marco se defiende la necesidad de trabajar en el aula la competencia sociolingüística (Instituto Cervantes, 2002: §5.2), a la vez que se efectúa una clara distinción entre la lengua artificial, objeto del aprendizaje, y la lengua natural, instrumento real de comunicación (cfr. supra, $\$ 1.1$ ), lo que constituye quizá per se un argumento para llevar ambos aspectos al aula.

En efecto, entre los motivos para hacer explícita la distinción entre lengua natural y lengua artificial cabe destacar que los malentendidos que pueda sufrir el alumno, basados en las expectativas comunicativas creadas sobre las características de la lengua artificial y no de la lengua natural, pueden conducirle a ciertos sentimientos de frustración si reconoce que «nadie habla así» o si percibe que tiene que corregir a los nativos porque «no hablan como en sus fichas» $\mathrm{o}$ «no dicen lo que el profesor me ha enseñado».

En cuanto a hacer explícita la diversidad lingüística, a la base metodológica del Marco se le puede añadir otros argumentos por los que visibilizar las lenguas de España en la clase de EL2, lo que entendemos que no se suele hacer en el aula, debido a su práctica inexistencia en los manuales (Fernández Martín, 2013c*87 ss).

En primer lugar, el hecho de que los materiales de los manuales Español lengua viva 1 y Nuevo Prisma (A1) ofrezcan breves explotaciones didácticas que toman como objeto de aprendizaje las lenguas cooficiales de España, viene a demostrar que se pueden enseñar estas lenguas en cualquier nivel de aprendizaje de ELE, 
siempre que se adapte, como corresponda, a las necesidades comunicativas y al nivel de lengua del alumnado (Fernández Martín, 2013c: 90-92). Asimismo, nuestra propia experiencia docente (cfr. $₫ 3$ ) demuestra que es tan posible como necesario hacerlo y que no implica ningún tipo de «trauma» para los alumnos, si se contextualizan adecuadamente y se acompañan de las explicaciones sociolingüísticas oportunas.

En segundo lugar, dado que la mayoría de los manuales contiene alguna unidad didáctica (o sección dentro de todas ellas) en que se trabajan la diversidad lingüística y cultural, los estilos individuales de aprendizaje o la situación del español en el mundo (Fernández Martín, 2013c: 88 ss), parece deducirse que cabría perfectamente una reflexión sociolingüística en las unidades didácticas adecuadas.

En tercer lugar, el hecho de que los estudiantes de EL2 residentes en España vivan en una ciudad teóricamente monolingüe como Madrid, no les impide viajar (por ocio o negocio) por todo el país, por lo que se van a encontrar de bruces, entre otras muchas lenguas, con el catalán, el gallego y el vasco, cuánto más si nos encontramos enseñando español en una región oficialmente bilingüe (Etxebarria, 2002), donde dichas lenguas son necesarias para mantener las relaciones sociales día a día y no conocerlas puede implicar condenarse a la exclusión social (Sabaté i Dalmau, 2008).

En cuarto lugar, conviene añadir la importancia de la identidad, de la que la lengua forma naturalmente parte, por lo que se muestra como herramienta de acercamiento, empatía y comprensión con el otro (Crystal, 2001: 104; May, 2003; McCarty et alii, 2008; Fernández Martín, 2013c; Mesthrie, 2008).

En quinto lugar, mostrar la diversidad lingüística puede entenderse como una manera de educar en valores, tratando de hacer explícita la reflexión sobre las desigualdades sociales (que son aceptadas como naturales), plasmadas en las variedades lingüísticas, las cuales a su vez se utilizan como herramientas simbólicas (de abuso) del poder (Bourdieu, 2008; McCarty et alii, 2008).

En este caso, las lenguas minoritarias desempeñarían un papel fundamental si fueran enseñadas en tanto lenguas, válidas per se, y no en tanto instrumento evaluado sociocríticamente $\left(\mathrm{cfr}_{+} \S 3\right)$ : al fin y al cabo, son herramientas de comunicación, creación de mundos, expresión de culturas, conformantes identitarios y medios de transmisión de conocimientos, exactamente en la misma medida que las lenguas mayoritarias (Crystal, 2001; McCarty et alii, 2008; Tusón, 2009; Moreno Cabrera, 2004).

Asimismo, si se entiende que el objetivo último de la enseñanza de idiomas es acercar al alumno al éxito comunicativo, ¿no resulta, pues, deseable hacerle ver 
que cuando una persona bilingüe se dirige a él en gallego en lugar de emplear el castellano lo está haciendo por cuestiones de identidad (es su lengua materna) o de costumbre (le sale sin pensar), y no porque no quiera ser entendido? Si en clase se ha reflexionado sobre ello, a lo mejor el estudiante de ELE deja de tomarse a mal dichos cambios de código tan típicos de los hablantes bilingües (Vinagre Laranjeira, 2005: 13-15; Salzmann, 2007: 173-195).

Finalmente, no cabe descartar, como en cierto sentido indica Crystal (2001: 69 ss), el aprendizaje de otras lenguas, sencillamente, porque sí, como objeto valioso en sí mismo (Tusón, 2009; McCarty et alii, 2008), contribuyendo a fomentar la competencia plurilingüe defendida en el Marco (Instituto Cervantes, 2002: $\$ \$ 1.3,6.1 .3)$.

Teniendo, pues, todas estas cuestiones en cuenta, nosotros sugerimos en la actividad diaria del aula, por un lado, la distinción explícita entre la lengua de enseñanza y la lengua de comunicación; y, por otro lado, la inclusión de las lenguas de España en la clase de ELE dentro de los contenidos socioculturales del currículo. En el siguiente apartado reflexionamos sobre ambas propuestas a partir de la experiencia docente.

\section{Sociolingüística española en el aula: análisis crítico de una propuesta didáctica}

Acerquémonos ahora a nuestra práctica docente para esgrimir la incorporación de las lenguas españolas al aula de EL2. A finales del año 2012, la inquietud de una alumna china de nivel B2 de español como lengua extranjera, ante una noticia televisiva de la que no entendía lo que decía uno de los hablantes (un político catalán que no hablaba en castellano), nos animó a adaptar una unidad didáctica creada para otro contexto (Fernández Martín, 2012), en la que se enseñaran, siquiera globalmente, las otras lenguas de España. El objetivo no era enseñarle a hablar catalán, gallego o vasco, lenguas que desgraciadamente la docente en cuestión no conocía, sino hacer llegar a esta estudiante y a sus compañeros que en nuestro país no se habla sólo castellano y que, en realidad, tampoco se hablan sólo las lenguas que se consideran oficiales, sino que las variedades existentes son casi tan amplias como el número de hablantes (recuérdese el concepto de idiolecto; Salzmann, 2007: 175), lo que implicaba mostrarles la diversidad sociolingüística de España, así como hacerles conscientes de la diferencia ideológica entre lengua estándar y lengua hablada.

La secuencia estaba pensada para realizarse en dos horas, dada la asistencia irregular de los alumnos para los que se concibió, si bien fue modificándose con 
el paso del tiempo (Fernández Martín, 2012, 2013c; cfr. anexos)ः en la primera fase, la unidad didáctica se llevó a cabo con alumnos inmigrantes en un centro de adultos situado en un barrio humilde del noroeste de Madrid capital; las sucesivas fases se aplicaron con alumnos de un perfil muy diferente, dado que eran todos trabajadores de clase media-alta que podían costearse un curso de muchas horas al mes en una escuela situada en el madrileño barrio de Salamanca.

Así pues, en una primera fase la secuencia estaba formada por actividades de distinto tipo y dificultad, desde un «unir con flechas» entre las palabras y la definición de conceptos como lingua franca, lengua común y lengua estándar o diglosia, variedad diafásica, variedad diastrática o variedad individual, hasta un «localiza las palabras de cada lengua en los textos», donde los alumnos comparaban algunas palabras del artículo 2.1 de la Declaración Universal de los Derechos Humanos, que se les presentaba en castellano, catalán, gallego, vasco y asturiano (Fernández Martín: 2012, 2013c; cfr. anexo I, actividades 2, 3; y anexo II, tareas 1, 2, 3, 5).

A continuación, se les proponía la comprensión de diversos fragmentos de textos en castellano sobre la diversidad lingüística, escritos por distintos expertos (Amin Maalouf, David Crystal, Juan Ramón Lodares, el Manifiesto por la lengua común), cuyo fin último era la creación de opinión para la tarea final, que era un debate en torno al lema «Nueva propuesta de leyः se prohíbe hablar otra lengua distinta al español en los lugares públicos». Se les explicó que habían sido convocados por el Senado para debatir el tema propuesto en calidad de expertos y debían dar su opinión sobre el tema. En un segundo grupo esto se modificó, y se pidió que cada alumno defendiera la opinión de uno de los autores que por sorteo les había tocado anteriormente en una interacción oral por parejas (Fernández Martín, 2012, 2013c; cfr. anexo I, actividades 4, 5; anexo II, tareas 6, 8, 9).

En una segunda fase de aplicación de la unidad didáctica, se procedió a trabajar con material procedente de manuales de ELE en los que había alusiones a las lenguas de España y que, incluso, ofrecían una muestra auditiva de cada una de ellas (Fernández Martín, 2013c), como son los ya citados Español lengua viva 1 (p. 24) y Nuevo Prisma (A1) (pp. 26-27).

En esta segunda fase, el nivel del grupo se amplió del B2 al A1, lo que implicaba la posibilidad de utilizar la unidad didáctica también en A2 y B1, haciéndola cada vez más flexible (eliminando el debate final y centrando el objetivo en adquisición de léxico y desarrollo de las destrezas perceptivas). Estas constantes modificaciones nacían de la firme convicción de que es posible concienciar lingüísticamente a cualquier nivel de la LE, siempre y cuando se propongan actividades adecuadas al nivel, como se defiende desde el enfoque por tareas (Martín, 1995; Ribé, 1997; Estaire, 1999; Pica, 2008). 
Así, en una siguiente etapa, se le añadió una actividad basada en la interesantísima propuesta docente del Grupo Eleuterio Quintanilla (2006: 49 ss). Tras pegar por las paredes de la clase el conjunto de textos ahí propuesto, formado por diversos poemas en algunas lenguas de España (catalán, euskera, gallego, asturiano y castellano) y las respectivas biografías de los autores, se les pidió que, en primer lugar, leyeran cada texto para relacionarlo con la lengua en que estaba escrito, ayudándose de la tabla realizada con los fragmentos de la Declaración Universal de los Derechos Humanos y, en segundo lugar, que trataran de averiguar quién había escrito qué, siguiendo naturalmente las pistas de las propias biografías de los autores.

Finalmente, en una última etapa se creó material más complejo (actividades sobre todo de léxico especializado y una comprensión auditiva sobre la base de un discurso pronunciado por la senadora Leire Pajín en el que utilizaba las lenguas cooficiales para defender la pluralidad lingüística del Estado), llevado a un grupo de nivel C (cfr. anexo I, actividades 3; anexo II, tareas 4, 6, 7), lo que en principio dio por cerrados nuestras intenciones y objetivos, si bien esta propuesta se encuentra, como cualquier otro material didáctico, en constante construcción.

Desde una perspectiva crítica, las tareas realizadas han dado, por lo general, buenos resultados. En el caso de los grupos de inmigrantes, cabe señalar una anécdota: después de acabar la secuencia de actividades que aquí hemos incluido dentro de la primera fase, dos alumnas arabófonas procedentes de distintos países acordaron durante unas semanas darse clase mutuamente de las lenguas que desconocían ( $A$ dio unas clases de inglés a B, B se las dio de francés a A), en una demostración de interés interlingüístico.

La segunda fase de la unidad didáctica se llevó a cabo con numerosos grupos de procedencias diversas, generalmente occidentales. Solían mostrar interés, dado que todos ellos habían visitado alguna comunidad autónoma con una lengua cooficial (en su mayoría, Cataluña, por la atracción que suponía la ciudad de Barcelona) y se habían encontrado con carteles que no entendían, lo que habían achacado a que no tenían una alta competencia en castellano. Esta deducción provocaba constantemente sentimientos de sorpresa ante la existencia de otras lenguas distintas al español, de las que en numerosas ocasiones ni siquiera habían oído hablar.

Después de hacer las actividades de esta fase y la tercera, confesaban con frecuencia que les resultaba muy curioso realizar las comprensiones auditivas de los manuales de ELE, ya que nunca habían oído conscientemente otras lenguas españolas, y se sorprendían cuando veían que, dada la contextualización de la 
actividad, entendían lo que se les pedía, con la única excepción del euskera, que siempre les costaba un poco más.

A este respecto, resulta reseñable el papel desempeñado por la lengua vasca en nuestras actividades, ya que, por un lado, era evidente la dificultad que les entrañaba pronunciar los textos e intentar «descifrar» su significado (incluso contando con la traducción más o menos literal) pero, por otro lado, los textos escritos (u oídos) en esta lengua eran los primeros que ellos clasificaban como tales. En otras palabras, no entendían lo que ponía en los textos vascos, pero les resultaba mucho más sencillo distinguir que se trataba de la lengua vasca que en el caso de las otras tres lenguas románicas trabajadas (asturiano, gallego y catalán), dejando de lado el castellano, que en principio les resultaba, dependiendo de los niveles de conocimiento, (un poco) más familiar.

Otro aspecto interesante, aunque esperable, fue descubrir lo difícil que es luchar contra los prejuicios de los estudiantes. A modo anecdótico, un alumno marroquí de $\mathrm{B} 1$ descartó rápidamente el texto en asturiano, durante la actividad de comprensión lectora (cfr. anexo I, act. 4; anexo II, act. 5), porque se le había dicho que no era una lengua oficial. «Si no es oficial no es lengua», llegó a afirmar, dejando así claramente entrever el valor del poder en la configuración sociocognitiva de las lenguas. Tener este hecho en cuenta supone un apoyo aún mayor a la necesidad de concienciar sobre la diversidad lingüística.

No hay que olvidar, por otra parte, que este tipo de actividades carecían, en el fondo, de una concienzuda labor de distinción entre la lengua estándar y la lengua oral, más allá de las reflexiones metalingüísticas que, especialmente con los grupos avanzados (en los iniciales resulta complejo entablar debate por su falta de recursos lingüísticos), tuvieron lugar sobre el papel de la norma, el cuestionamiento de la necesidad de una institución reguladora del idioma y la comparación intercultural de los prejuicios que había entre conceptos como lengua y dialecto, variedad estándar y variedad local o sobre las consecuencias de no estar alfabetizados.

Y este es el mayor de los puntos débiles de nuestra unidad didáctica: a pesar de haber sido concebida desde una perspectiva sociolingüística, centrada en el conocimiento de las lenguas de España, se dejó de lado el trabajar explícitamente la diferencia entre la lengua estándar, que es la lengua que el alumno estudia, especialmente en los primeros niveles de aprendizaje (cfr. $₫ 1$ ), y las variedades reales que cualquier extranjero se va a encontrar en el día a día, cronotópicamente localizadas. Se creyó que sería suficiente con alguna actividad de léxico en la que, simplemente, se hiciese alusión a distinciones terminológicas. Y, sin embargo, llegamos a la conclusión de que esto no bastaba para concienciar a los estudiantes 
de la diferencia entre lengua natural y lengua artificial ya que, en realidad, no habíamos diseñado ninguna tarea ex profeso para ello.

Parece evidente, además, que la incorporación implícita en los manuales de ELE de acentos o vocabulario de todo el mundo hispano, distintos al del español de España, no servía de mucho cuando numerosos alumnos, sobre todo en niveles intermedios (lo que ya supone un conjunto considerable de horas de dedicación al estudio de la LE), no valoraban la complejidad intralingüística de cualquier variedad. Es decir, dado que no se les consciencia realmente de que cualquier variedad de la lengua es igual en tanto herramienta comunicativa, siempre hay un halo de superioridad hacia la lengua estándar como perfecta (y única) para comunicarse, lo que mantiene sus prejuicios a lo largo de todo el proceso de aprendizaje, llevándoles así, en ocasiones, a un sentimiento de fracaso en las interacciones con los nativos. Y mucho menos se da, como vemos, esta concienciación con relación a otras lenguas que conviven con el castellano.

\section{Conclusiones}

El objetivo de este artículo, que podemos enmarcar dentro de la educación lingüística, consistía en defender la distinción explícita en el aula entre los conceptos de lengua artificial y lengua natural, entendidos en el Marco como «lengua estándar» $y$ «dialecto», respectivamente; $y$ la visibilización de las lenguas de España en la clase de ELE, cuyo desconocimiento puede implicar serios problemas interaccionales entre los extranjeros y los nativos.

En efecto, tras la revisión a nuestra propuesta didáctica, parece haber quedado claro, por sus carencias, que existe la necesidad de que los estudiantes sean conscientes de la diferencia entre lengua de aprendizaje (lengua artificial) y lengua de comunicación (lengua natural), lo que obligatoriamente pasa por la creación de actividades que conlleven la reflexión sobre lo que estudian en el aula y lo que se encuentran luego en la calle, especialmente si se trata de estudiantes de EL2. Dada la complejidad del nivel de lengua que se precise para debatir temas tan espinosos, quizá en este caso sea conveniente un conocimiento mínimo por parte del alumno de la LE para poder tratarlo en clase (principios de A2), si bien conviene hacerlo cuanto antes, ya que cualquier malentendido comunicativo en niveles inferiores será probablemente interpretado por el estudiante de ELE desde su ignorancia de la lengua. Esto equivale a decir que los estudiantes de EL2 que se encuentren en niveles iniciales tenderán a explicar las diferencias entre la lengua artificial y la lengua natural acudiendo a su propio desconocimiento de la L2, no en base a que realmente sean conscientes de que se trata de dos variantes distintas. 
Por otra parte, que se haya propuesto la enseñanza de la diversidad lingüística española no impide que se efectúe también en la enseñanza del EL2 en otros países hispanohablantes, donde el castellano convive con lenguas como el náhuatl, el quechua o el aimara, cuyas principales nociones también convendría dar a los estudiantes extranjeros; ni en la enseñanza de otros idiomas, como el inglés, el francés o el alemán, cuyas lenguas objeto también comparten territorio con otras lenguas.

Entendemos, por tanto, que lo relevante es contribuir a la formación de usuarios competentes de la LE, desde una perspectiva lingüística, por supuesto, pero también desde una perspectiva sociolingüística y pragmática, que se traduzca, en la práctica, en la adquisición de una alta competencia plurilingüe.

\section{Referencias bibliográficas}

(Todas las páginas web han sido revisadas el 25 de febrero de 2014.)

Bourdieu, P. (2008). ¿Qué significa hablar? Economía de los intercambios lingüísticos. Madrid: Akal.

Caballero Murillo, M; y Cuadrado Gordillo, I. (2007). «La sociolingüística y la enseñanza de la lengua y la cultura de origen». Revista de Educación, 342: 503-527.

Campero, C. (2007). «Puntos de referencia internacional sobre la alfabetización de adultos». Transatlántica de educación, 2: 22-2.

Chambers, J. K. (I995). Sociolinguistic Theory. Oxford: Blackwell.

Cots, J. M. et alii. (2007). La conciencia lingüistica en la enseñanza de lenguas. Barcelona: Graó.

Crystal, D. (2001). La muerte de las lenguas. Madrid: CUP.

EACEA (2011). La educación formal de adultos en Europa: politicas y prácticas. Bruselas: Eurydice España/MEC.

Duranti, A. (2000). Antropología lingüística. Madrid: CUP.

Estaire, S. (1999). Tareas para hacer cosas en español. Principios y práctica de la enseñanza de lenguas extranjeras mediante tareas. Madrid: Fundación Universidad Antonio de Nebrija.

Etxebarria, M. (2002). La diversidad de lenguas en España. Madrid: Espasa.

FASOLd, R. (1996). La sociolingüistica de la sociedad. Introducción a la sociolingüistica. Madrid: Visor.

Fernández Martín, P. (2008): «Fonética para inmigrantes: la necesidad de conocer el sistema fonológico de la LM del alumno». En: A. Moreno SandovAL (ed.) (2008): El valor de la diversidad [meta]lingüística (Actas VIII Con- 
greso de Lingüística General, celebrado en Madrid los días 25-28 junio de 2008). Madrid: Universidad Autónoma: 655-674. [<www.lllf.uam.es/clg8/ actas/pdf/paperCLG35.pdf > ]

Fernández Martín, P. (2012). Gotas de antropología para anclarnos en la red existencial. Saarbrücken: EAE.

- (2013a): «Alfabetizando arabófonos adultos: un estudio de casos». En: Revista Nebrija de Lingüistica Aplicada, 13 (número especial - Actas del Congreso En camino hacia el plurilingüismo, celebrado en Madrid los días 28 a 30 de septiembre de 2012). [<http://www.nebrija.com/revista-linguistica/files/ revistasPDF/526a47dc03a6e_revista_completa_13.pdf $>$ ]

- (2013b). «El papel de las canciones en la enseñanza de idiomas a inmigrantes: una propuesta didáctica». MarcoELE. Suplementos, 17, julio-diciembre. [<http $/ /$ marcoele.com/suplementos/canciones-a-inmigrantes/ $>$ ]

- (2013c). «El papel de las otras lenguas en la clase de español». Revista de Filoloxía Asturiana, 13: 71-106. [<http://www.unioviedo.es/reunido/index. php/RFA/article/view/10259/9895>]

Ferreiro, E. (comp.) (2002). Relaciones de (in)dependencia entre oralidad y escritura. Barcelona: Gedisa.

Grupo Eleuterio Quintanilla (2006). Lengua y diversidad cultural. Actividades para el aula. Madrid: Talasa.

Heller, M. S. (1982). «Negotiations of language choice in Montreal». En J. J. Gumperz (ed.) (1982). Language and social identity. Cambridge. CUP: $108-118$.

Instituto Cervantes (2002). Marco común europeo de referencia para las lenguas: aprendizaje, enseñanza, evaluación. Madrid: MECD/Anaya.

Jupp et alii. (1982). «Language and disadvantage: the hidden process». En: J. J. Gumperz (ed.) (1982). Language and social identity. Cambridge. CUP: 232-256.

Lodares, J. R. (2000). El paraíso políglota. Madrid: Taurus.

López Morales, H. (1989). Sociolingüística. Madridः Gredos.

Lyovin, A. V. (1997). An Introduction to the Languages of the World, Nueva York: Oxford University Press.

Maflouf, A. (2004). Identidades asesinas. Madrid: Alianza.

Martín Peris, E. (1995). «Las tareas en la enseñanza de E/LE a adultos». En: M. Siguán (ed.). La enseñanza de la lengua por tareas. Barcelona. Universidad de Barcelona: 39-56.

Martín Rojo, L. (1994). "The jargón of delinquents and the study of conversational dynamics», Journal of Pragmatics, 21: 243-289. 
Martínez-Otero Pérez, V. (2008). El discurso educativo. Madrid: CCS.

May, S. (2003). «Rethinking Linguistic Human Rights. Answering Questions of Identity, Essentialism and Mobility». En: D. Patrick y J. Freeland (eds.): Language Rights and Language 'Survival': A Sociolinguistic Exploration. Manchester. St. Jerome Publishing*35-53.

McCarty, T. et alii. (2008)+ «Education for Speakers of Endangered Languages». En: B. Spolsky y F. M. Kult (eds.). The Handbook of Educational Linguistics. Singapur. Blackwell: 297-312.

Mesthrie, R. (2008). "Sociolinguistics and Sociology of Language». En: B. Spolsky y F. M. Kult (eds.). The Handbook of Educational Linguistics. Singapur. Blackwell: 66-82.

Moreno Cabrera, J. C. (2004). La dignidad e igualdad de las lenguas. Crítica de la discriminación lingüística. Madrid: Alianza Editorial.

- (2005). La lengua y sus escrituras. Tipología, evolución e ideología. Madrid: Síntesis.

- (2008): El nacionalismo lingüistico. Una ideología destructiva. Barcelona: Península.

- (2014). Los dominios del español. Guía del imperialismo lingüístico panhispánico. Madrid: Euphonia Ediciones.

Moreno Fernández, F. (2007). «Adquisición de segundas lenguas y Sociolingüística». Revista de Educación, 343: 55-70.

Pica, T. (2008). «Task-Based Teaching and Learning». En: B. Spolsky y F. M. Kult (eds.). The Handbook of Educational Linguistics. Singapur. Blackwell: 525-538.

Rıbé, R. (1997). «El procesamiento de lengua». En R. Ribé y M. L. Celaya (eds.) (1997). Tramas creativas y aprendizaje de lenguas. Prototipos de tareas de tercera generación. Barcelona. Universidad de Barcelona: 95-105.

Richards J. C. y Rodgers, T. S. (2003). Enfoques y métodos en la enseñanza de idiomas. Cambridge: CUP.

Sabaté i Dalmau, M. (2008). «Inmigración y multilingüismoः cambios en el mercado lingüístico y la categorización social de (in)migrantes en una localidad periférica de Barcelona». En* A. Moreno Sandoval (ed.) (2008): El valor de la diversidad [meta]lingüística (Actas VIII Congreso de Lingüística General, celebrado en Madrid los días 25-28 junio de 2008). Madrid: Universidad Autónoma: 1746-1765 [<www.lllf.uam.es/clg8/actas/ActasCLG8. pdf $>$ ].

Salzmann, Z* (2007). Language, Culture and Society. Oxford: Westview Press. 
Sánchez, A. (2000). Los métodos en la enseñanza de idiomas. Evolución bistórica $y$ análisis didáctico. Madrid: SGEL.

Tusón Valls, A. y Calsamiglia Blancáfort, H. (1999). Las cosas del decir. Manual de análisis del discurso. Barcelona: Ariel.

Tusón, J. (2009). Patrimonio natural. Elogio y defensa de la diversidad lingüística, Barcelona: Ariel.

Velasco, H. y Díaz de Rada, Á. (2006). La lógica de la investigación etnográfica. Un modelo de trabajo para etnógrafos de escuela. Madrid: Trotta.

Vinagre Laranjeira, M. (2005). El cambio de código en la conversación bilingüe: la alternancia de lenguas. Madrid: Arco/Libros.

ZANóN, J. (2007). «Psicolingüística y didáctica de las lenguas: una aproximación histórica y conceptual». marcoELE. Revista de Didáctica ELE, 5 [<httpः// www.marcoele.com/num/5/02e3c099650f54607/psicolinguistica.pdf $>$ ]

\section{Material didáctico citado}

Equipo nuevo Prisma (2012), Nuevo Prisma. Curso de español para extranjeros. A1, Madrid: Edinumen.

Susana Gómez y Ma Antonia Oliva (eds.) (2008). Español lengua viva 1. A1A2, Madrid: Santillana.

\section{Webgrafía}

Declaración Universal de los Derechos Humanos: <http://www.ohchr.org/EN/ UDHR/Pages/Introduction.aspx>

Real Academia Española (2001). Diccionario de la lengua española (22.a ed.). Consultado en <http://www.rae.es/rae.html>

«Manifiesto por la lengua común»-El País, 23 de junio de 2008. Web: <http:// elpais.com/elpais/2008/06/23/actualidad/1214209045_850215.html> 


\section{Anexo I. Actividades para trabajar la diversidad lingüística}

\section{Actividad 1. Motivación y sondeo de conocimientos}

Objetivo: Motivar y sondear conocimientos

Duración: 5 minutos

Dinámica: grupo-clase

Nivel: B (B1-B2)

Material: un mapa de España (o de la zona geográfica que sea oportuna)

Instrucciones: se les presenta un mapa de España y se les pregunta qué conocen del país. Si han estado en comunidades autónomas bilingües, se les pregunta si notaron algo raro en la forma de hablar de la gente.

\section{Actividad 2. Introducción a la sociolingüística}

Objetivo: presentar léxico específico

Duración: 30 minutos

Dinámica: individual

Nivel: B

Material: tareas 1 y 2 del anexo II

Instrucciones: cada alumno debe unir con flechas los conceptos de la columna de la izquierda con la definición de la columna de la derecha (tarea 1). A continuación, debe emplear esos mismos conceptos para completar el texto (tarea 2).

\section{Actividad 3. Profundización en sociolingüística}

Objetivo: ampliar el léxico específico

Duración: 15 minutos

Dinámica: individual, por parejas

Nivel: C (C1-C2)

Material: tareas 3 y 4 del anexo II

Instrucciones: cada alumno debe intentar adivinar a qué concepto hace alusión cada una de las definiciones expuestas (tarea 3). Una vez corregido, con ayuda del compañero, cada estudiante intentará relacionar dicha definición con el ejemplo lingüístico correspondiente (tarea 4). 


\section{Actividad 4. Presentación de otras lenguas peninsulares}

Objetivo: presentar otras lenguas peninsulares

Destrezas: comprensión lectora, expresión oral

Duración: 20 minutos

Dinámica: parejas

Nivel: B (B1-B2)

Material: tarea 5 del anexo II

Instrucciones: presentar el texto (por ejemplo, el artículo 2.1 de la Declaración Universal de los Derechos Humanos; cfr. anexo III) en castellano y el correspondiente en las demás lenguas (por ejemplo, catalán, gallego, euskera, asturiano). Pedirles a los alumnos que completen una tabla con los sinónimos interlingüísticos. Se le puede dar unos textos al alumno A de la pareja, otros al alumno B y que completen la tabla juntos. Se les puede preguntar sobre semejanzas y diferencias. ¿Qué lenguas pondrían juntas?

Si el grupo es lingüísticamente homogéneo, se les puede pedir que hagan una primera fase de búsqueda y familiarización con su lengua materna ( $y$ las de esa familia lingüística), para después trabajar el español y las lenguas afines (por genealogía o por geografía) a este.

\section{Actividad 4. Tarea final: debate}

\subsection{Adquisición de opiniones}

Objetivo: dotar a los alumnos de diversas opiniones sobre el multilingüismo

Destrezas: comprensión lectora, comprensión auditiva, expresión oral

Duración: 40 minutos (CL, EO) + 20 minutos (CA)

Dinámica: «bola de nieve»

Nivel: B (B1-B2). La audición funciona mejor en el nivel C.

Material: tareas 6 y 7 del anexo II; textos del anexo IV

Instrucciones: se divide la clase en grupos de cuatro. A cada grupo se le entrega un texto (multiplicado por el número de alumnos de que esté formado). Se les deja unos 10 minutos para leerlo, comprenderlo y rellenar la fila correspondiente en la tabla. A continuación, se les empareja A-B y C-D (o A-C y B-D), de forma que cada alumno complete, con ayuda del compañero, otra fila de la tabla. Finalmente, se les agrupa A-B-C-D para terminar de completar la tabla. Una puesta en común permitirá a los alumnos corregir a sus propios compañeros de grupo en caso de que no hayan entendido bien los textos. El profesor terminará revisando que los contenidos de la tabla sean correctos. 
En una segunda fase (tarea 7), se les presenta una fotografía de la política Leire Pajín y se les pregunta si la conocen. Se les dice que van a escuchar qué dijo en el Senado el 28 de abril de 2010. Podrán oírla hasta tres veces, dada la complejidad de la intervención: <http://www.youtube.com/watch?v=Nhcl6OEXtlE $>$ (Min. 3'35"-7'10").

\subsection{Reflexión sobre lo visto}

Objetivos: reflexionar sobre todo lo leído y escuchado

Destrezas: interacción oral

Duración: 20 minutos

Dinámica: parejas

Nivel: B (B1-B2)

Material: tarea 8 del anexo II; textos del anexo IV

Instrucciones: A cada pareja se le entrega un par de las preguntas sobre los textos, ya leídos y comentados previamente, para profundizar en su contenido. Tienen que responderlas de forma oral.

\subsection{Debate final}

Objetivos: ordenar las ideas de lo estudiado y expresarlas de forma oral

Destrezas: expresión e interacción oral

Duración: 20 minutos

Dinámica: grupos; grupo-clase

Nivel: B (B1-B2)

Material: textos expuestos en el anexo IV (y utilizados en las tareas 6 y 8 )

Instrucciones: Se divide la clase en grupos. A cada alumno se le entrega una tarjeta con el nombre del autor (de los textos vistos) que debe representar. Se escribe en la pizarra lo siguiente: «Nueva propuesta de ley: se prohíbe hablar otra lengua distinta al español en los lugares públicos». Han sido convocados por el Senado para debatir el tema propuesto en calidad de expertos y deben dar su opinión según el autor que les ha tocado.

Variante: si se considera necesario, puede abrirse el debate al grupo-clase después de unos minutos de debate por grupos. Puede hacerse apelando a opiniones más subjetivas, como las mostradas para realizar la siguiente actividad. 


\section{Actividad 5. Texto argumentativo}

Objetivos: ordenar las ideas de lo estudiado y expresarlas por escrito

Destrezas: expresión escrita

Duración: 30 minutos

Dinámica: individual

Nivel: B (B1-B2)

Material: tareas anteriores y textos del anexo IV

Instrucciones: Cada alumno, como ejercicio extra, tiene que escribir un texto argumentativo (150-200 palabras) en el que exprese su propia opinión sobre el tema aquí tratado.

Estructura posible: introducción (tipo de enfoque: general — todas las lenguas-, particular — una lengua en concreto-), argumentos (a favor, en contra, soluciones), conclusión (¿Complejidad o sencillez?)

\section{Anexo II. Material didáctico}

Se incluyen las soluciones directamente en el material. La dinámica de cada actividad se ha explicado en el anexo anterior.

Tarea 1. Relaciona cada palabra con su definición:

\begin{tabular}{|l|l|}
\hline Lingua franca & Lengua que sirve como instrumento de comunicación internacional. \\
\hline Lengua propia & $\begin{array}{l}\text { Lengua que se considera típica de una región, pero no de otras regiones ve- } \\
\text { cinas o del resto del país. }\end{array}$ \\
\hline Lengua oficial & $\begin{array}{l}\text { Lengua que se usa en contextos jurídicos (documentos, leyes, inscripciones, } \\
\text { contratos....). }\end{array}$ \\
\hline Lengua común & Lengua que se entiende como compartida por una comunidad de hablantes. \\
\hline
\end{tabular}

Tarea 2. Lee el texto y complétalo utilizando estas expresiones (pueden estar en plural): lengua propia ( $x$ 2) - lengua común - lengua oficial (x 3) - lingua franca

\section{¿Español o castellano?}

En el ámbito de la política lingüística resulta frecuente encontrar debates sobre la denominación de la lengua oficial de España.

Desde el punto de vista legal, la Constitución española de 1978 afirma que la

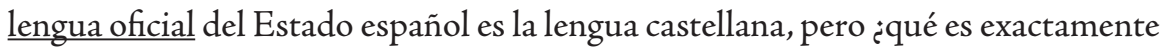
la «lengua castellana»? 
Algunos expertos defienden que llamarla «castellano» puede llevar a confusión porque tiene al menos tres significados: $a$ ) el dialecto hablado en Castilla; $b$ ) la misma lengua española y $c$ ) la lengua española medieval.

Estos mismos estudiosos creen que por ello resulta más oportuno denominarla «lengua española», ya que si bien puede no ser la lingua franca (una lengua de comunicación internacional), sí puede funcionar como una lingua franca, ya que el español es una lengua hablada por millones de personas.

Sin embargo, la opinión contraria sostiene que las lenguas propias de determinadas Comunidades Autónomas (catalán, gallego, vasco) son también «lenguas españolas», y que por ello denominar «español» al castellano puede ser discriminatorio para los hablantes de dichas lenguas. Asimismo, aunque haya países donde la lengua adquiere el nombre de la nación («francés», «italiano»), también los hay en los que su principal lengua oficial es denominada en función del origen de dicha lengua (por ejemplo, «inglés» en vez de «británico»o «ruso» en vez de «soviético»).

La más reciente solución pasa por considerar «lengua española» a la lengua común a todo el país cuando se enfrenta a las lenguas extranjeras («español» frente a «portugués»), pero llamarla «lengua castellana» cuando se pretende diferenciarla de las lenguas propias de determinadas Comunidades Autónomas.

Tarea 3. Escribe cada una de los siguientes conceptos junto a la definición que creas adecuada: A) diglosia; B) variedad diafásica o de registro; C) lengua estándar; D) variedad diastrática o sociolecto; $\mathrm{E}$ ) variedad individual o idiolecto; F) bilingüismo; G) alternancia de códigos; H) lingua franca; I) normalización; J) criollización (Definiciones adaptadas a partir de DRAE; Salzmann, 2007; Chambers, 1995; Fasold, 1996; López Morales, 1989).

\begin{tabular}{|l|c|}
\hline $\begin{array}{l}\text { 1. Variedades de una lengua que se localizan en las hablas típicas de diferentes ámbitos } \\
\text { sociales, como el habla de los pícaros. }\end{array}$ & D \\
\hline $\begin{array}{l}\text { 2. Variedades lingüísticas que se usan en las distintas situaciones sociales, como el habla } \\
\text { culta frente a la coloquial. }\end{array}$ & B \\
\hline 3. Hablas de individuos determinados con sus peculiaridades idiosincrásicas. & E \\
\hline $\begin{array}{l}\text { 4. Variedad lingüística que sirve como modelo a todos los hablantes de una lengua y } \\
\text { funciona, por tanto, como norma y sistema unificador. }\end{array}$ & C \\
\hline $\begin{array}{l}\text { 5. Situación en la que, en una comunidad lingüística multilingüe, cada lengua se emplea } \\
\text { con funciones comunicativas distintas, y una de ellas se considera socialmente superior a } \\
\text { las demás. }\end{array}$ & A \\
\hline 6. El uso de más de una lengua durante un mismo acto de habla o conversación. & G \\
\hline $\begin{array}{l}\text { 7. Fenómeno individual y social que consiste en el empleo de dos lenguas como medio de } \\
\text { comunicación indistintamente. }\end{array}$ & F \\
\hline
\end{tabular}




\begin{tabular}{|l|c|}
\hline 8. Lengua que sirve como instrumento de comunicación internacional. & H \\
\hline $\begin{array}{l}\text { 9. Proceso sociopolítico que consiste en convertir una variedad lingüística en lengua } \\
\text { estándar mediante la elaboración de ortografías, gramáticas y diccionarios, y a través de la } \\
\text { educación y de los medios de comunicación. }\end{array}$ & I \\
\hline $\begin{array}{l}\text { 10. Proceso por el cual una lengua pidgin, creada por dos comunidades lingüísticas para } \\
\text { comunicarse entre sí, se convierte en lengua materna de una comunidad. }\end{array}$ & $\mathrm{J}$ \\
\hline
\end{tabular}

Tarea 4. Lee los siguientes textos y señala qué términos de la actividad anterior ejemplifica cada uno. Añade, además, estas expresiones en los texto que consideres oportuno: $\mathrm{K}$ ) transferencia y $\mathrm{L}$ ) variedad diatópica o dialecto (pueden repetirse).

\section{Texto 1. [d,e]}

A (pícaro): Nada, que no, yo entrifo ya de día

(Martín Rojo, 1994: 259).

Texto 2. [ $\underline{\mathrm{b}, \mathrm{d}}]$

A: ${ }_{i}$ Hola Maríaaaa!

B: ${ }_{¡}$ Hola Juani! ¿Cómo andas?

A: Aquí, aguantando el chaparrón. ¿Qué te pongo?

B: Pues medio kilo de las naranjas estas tan fresquitas.

A: Uy, estas están riquísimas, eh... que ayer me llevé yo unas y, bueno, para chuparse los dedos, de verdad que sí.

\section{Texto 3. $[\mathrm{j}(\mathrm{f}, \mathrm{g})]$}

Long taim (time) bifo (before), wan-pela ailan (island), drai pela pik (pig) i save stap ya, na em i save kaikai ol (all) man (men) («Hace mucho tiempo, vivían en una isla grandes cerdos que solían comer personas». Tok- pisin, Nueva Guinea; Lyovin, 1997: 427).

\section{Texto 4. [l,e]}

Mare, me jeché arena zobre la quemaúra. Te yamé, te yamé deb de er camino... ¡Nunca $e^{b} t u v o \ldots$... e tó tan $z o l o ! ~ L a^{b}$ yama me comían, mare, y yo te yamaba, y tú nunca venía (Juan Ramón Jiménez, «La carbonerilla quemada»).

\section{Texto 5. [i]}


El contenido de la vigésima segunda edición del Diccionario de la lengua española publicado por la Real Academia Española fue profundamente revisado durante los nueve años transcurridos desde que, en 1992, apareció la versión anterior (RAE).

Texto 6. $[\underline{\mathrm{b}, \mathrm{f}, \mathrm{g}, \mathrm{k}}]$

(En un bar, al cobrar; $\mathrm{A}=$ camarera joven; $\mathrm{B}=$ cliente varón adulto.)

A: Una llesca [tostada] tens. Una llesca... oi era? [Una tostada era..+, ¿no?]

B: Una llesca. Un tubo de cerveza y un Marie Brizard con hielo.

A: I un qué?

B: Marie Brizard con hielo.

A: Una..+ ¿Una cerveza o un tubo?

B: Una...

A (le interrumpe): Un tubo, un tubo, sí. Cinc noranta [cinco noventa].(Cuenta el dinero, monedero en mano.)

B: Sí, espera, espera, que ya tengo suelto y no me tienes que dar tanto cambio...

A: Ah, vale.

B: Toma, sí. (Intercambio de monedas; sonido de máquina registradora.) I deu i això també [Adiós y esto también]. (Dándole propina.)

A: Venga, hasta luego (Extraído de Sabaté i Dalmau, 2008).

Tarea 5. Completa la tabla siguiendo el ejemplo, después de leer los textos que te da el profesor (cfr. anexo III).

Art. 2.1. Toda persona tiene los derechos y libertades proclamados en esta Declaración, sin distinción alguna de raza, color, sexo, idioma, religión, opinión política o de cualquier otra índole, origen nacional o social, posición económica, nacimiento o cualquier otra condición.

\begin{tabular}{|c|c|c|c|c|}
\hline Castellano & Català & Galego & Euskara & Asturiano \\
\hline toda persona & tothom & toda persoa & gizaki orori & toa persona \\
\hline raza & raça & raza & arraza & raza \\
\hline color & color & cor & larru-kolorea & color \\
\hline idioma & llengua & idioma & hizkuntza & llingua \\
\hline sexo & sexe & sexo & sexua & sexu \\
\hline religión & religió & relixión & erlijioa & relixón \\
\hline opinión política & opinió política & opinión política & politikako iritzia & opinión política \\
\hline $\begin{array}{c}\text { posición } \\
\text { económica }\end{array}$ & fortuna & $\begin{array}{c}\text { posición } \\
\text { económica }\end{array}$ & ekonomi maila & $\begin{array}{c}\text { posición } \\
\text { económica }\end{array}$ \\
\hline nacimiento & naixement & nacemento & jaiotza & nacencia \\
\hline
\end{tabular}


¿Hay algún parecido entre estas lenguas? ¿Alguna se diferencia de las demás? ¿Por qué crees que es así?

Tarea 6. Lee el texto que te da la profesora. Completa la columna de la tabla según corresponda con la opinión de cada autor.

\begin{tabular}{|l|c|c|l|}
\cline { 2 - 4 } \multicolumn{1}{c|}{} & Monolingüismo & Multilingüismo & Argumento principal \\
\hline Manifiesto & $\mathrm{X}$ & & Lengua común \\
\hline Amin Maalouf & & $\mathrm{X}$ & Identidades (compatibilidad) \\
\hline David Crystal & & $\mathrm{X}$ & $\begin{array}{l}\text { Cada uno (monolingüe) defiende } \\
\text { su lengua }\end{array}$ \\
\hline Juan Ramón Lodares & $\mathrm{X}$ & & Lenguas de las mayorías \\
\hline Leire Pajín & & $\mathrm{X}$ & Porque se hablan en la calle \\
\hline
\end{tabular}

Tarea 7. Escucha a la Senadora en el Congreso el 28 de abril de 2010. ¿Defiende el monolingüismo o el multilingüismo? ¿Cuál es su argumento principal?

Tarea 8. Habla con tu compañero de los temas que te da el profesor:

- Manifiesto. ¿Cuál es la lengua común a la que se hace referencia? ¿Por qué los autores del manifiesto defienden que esta lengua debe ser conocida por todos los españoles? ¿Cuáles son sus argumentos?

- David Crystal. ¿Qué solución propone este experto?

- Amin Maalouf. ¿Qué soluciones ofrece el autor? ¿Son posibles? ¿Cuál es su principal argumento?

+ J.R. Lodares. ¿Qué piensa este autor del multilingüismo? ¿Cuál es su argumento?

- Leire Pajín. ¿Cuál es la idea fundamental de la senadora? ¿Qué puede implicar en la práctica? ¿Cómo reacciona la Cámara a su sugerencia? ¿Dónde quedan las lenguas no oficiales?

Tarea 9. Y tú, ¿qué piensas? ¿En tu país se habla sólo una lengua? ¿Se enfadan algunos si se hablan más? ¿Por qué crees que es así? 


\section{Anexo III. Textos Declaración universal de los derechos bumanos}

2.1. Tothom té tots els drets i llibertats proclamats en aquesta Declaració, sense cap distinció de raça, color, sexe, llengua, religió, opinió política o de qualsevol altra mena, origen nacional o social, fortuna, naixement o altra condició.

2.1. Toa persona goza de tolos drechos y llibertaes afitaes nesta Declaración, ensin estremamientu nengún de raza, de color, de sexu, de llingua, de relixón, d'opinión política o de cualesquier otra mena, d'orixe nacional o social, de posición económica, de nacencia o de cualesquier otra condición.

2.1. Gizaki orori dagozkio Aldarrikapen honetan adierazitako eskubide eta askatasunak, eta ez da inor bereziko arraza, larru-kolorea, sexua, hizkuntza, erlijioa, politikako edo bestelako iritzia, sorterria edo gizarteko jatorria, ekonomi maila, jaiotza edo beste inolako gorabeheragatik.

2.1. Toda persoa ten os dereitos e liberdades proclamados nesta Declaración, sen distinción ningunha de raza, cor, sexo, idioma, relixión, opinión política ou de calquera outra índole, orixe nacional ou social, posición económica, nacemento ou calquera outra condición.

\section{Anexo IV. Textos para el debate}

[... A Aquellos que claman a favor de una única lengua mundial suelen proceder de naciones monolingües, y tienden a dar por hecho que, cuando llegue el día, será por supuesto su propia lengua la que hablará todo el mundo. Los problemas surgen cuando por razones religiosas, nacionalistas u otras, el voto se orienta en distintas direcciones, como siempre ha sucedido [... . Es perfectamente posible y muy recomendable pensar en un mundo donde todos hablen al menos dos lenguas: su propia lengua étnica y una lengua franca internacional. Puesto que las dos lenguas tienen distintos propósitos, uno orientado a la identidad y el otro a la inteligibilidad, no tienen por qué entrar en conflicto.

David Crystal (2001: 42, 43) 
Resulta difícil de entender en las «normalizaciones» lingüísticas que hoy están de moda la necesidad que tienen ciertos grupos de convertir a todos a su credo. Está bien que a uno le dé por cultivar una lengua minoritaria, pero si otro ni la tiene, ni la necesita y, a lo mejor, ni le ve el gusto o el beneficio a hablarla, ¿para qué va a convertirse a ella? [...] Cuando tal minoría [...] no entiende que compite con una lengua -el español- que no es minoritaria, se dedica a la conversión de almas descarriadas que viven en la oscuridad monolingüe de la lengua española y viven razonablemente bien en las tinieblas [... ]. Es en ese momento cuando la minoría empieza a resultar un poquito pesada.

J. R. Lodares $(2000 \div 269,272)$

Todas las lenguas oficiales en el Estado son igualmente españolas y merecedoras de protección institucional como patrimonio compartido, pero sólo una de ellas es común a todos, oficial en todo el territorio nacional y por tanto sólo una de ellas - el castellano- goza del deber constitucional de ser conocida.

Fragmento del Manifiesto por la lengua común (El País, 23/06/2008)

Conservar la lengua propia [... ] para que los que la hablan no se vean obligados a abandonarla si quieren acceder a lo que les propone la civilización actual; generalizar sin reservas la enseñanza del inglés, tercera lengua, explicándoles sin descanso a los jóvenes hasta qué punto es a la vez necesaria e insuficiente; fomentar, al mismo tiempo, la diversidad lingüística, tender a que haya en cada país mucha gente que domine el español, el francés, el portugués, el alemán, y también el árabe, el japonés, el chino y otras cien lenguas en las que la especialización es más infrecuente, y por tanto más valiosa tanto para el interesado como para la colectividad; tal me parece que es el camino de la sensatez para quien desee extraer del formidable desarrollo de las comunicaciones un enriquecimiento a todos los niveles [...].

Amin Maalouf (2004: 151) 